\title{
Pharmacological agents in post stroke recovery
}

\begin{abstract}
Stroke is the second commonest cause of death and disability in the world. In spite of the Amazonian advances in understanding of the pathophysiology and pathogenesis of stroke, there is unfortunately limited number of agents available in the armamentarium to fight stroke. There is therefore a compelling need to develop novel therapies to target restoration of the damaged brain and not merely protect or treat in the acute phase of the disease. By developing interventions which can be administered several days or weeks after the onset of stroke, which can essentially remodel the intact brain so as to compensate for the infarction, many more patients can be treated and brought back to their previous level of integration into the society as useful members. A variety of therapeutic approaches that could be considered Neuroprotective and Neurorestorative are currently in preclinical and clinical trials after stroke. There are essentially two varieties of restorative approaches. One is cell-based and includes stem cell transplantation with and without augmentation with growth factor and other variety is the pharmacological approach. Since the current review deals only with the later, we will deliberate on the several strategies currently being explored under this banner which include statins, erythropoietin and analogs, human chorionic gonadotropin, growth factors, and agents that increase cyclic GMP. Recent published literature was searched using PubMed and Google for the article reporting on Pharmacological approaches in Post stroke recovery.
\end{abstract}

Keywords: Stroke; Neurorestoration; Pharmacological agents
Volume I Issue 6 - 2014

\author{
MV Padma Srivastava,' Ashu Bhasin,' Tanu \\ Talwar,' Majaz Moonis ${ }^{2}$ \\ 'Department of Neurology, AllMS, India \\ 2Department of Neurology, UMass Memorial medical Centre \\ Worcester, USA
}

\begin{abstract}
Correspondence: Majaz Moonis, Department of Neurology, UMass Memorial Medical Centre Worcester, Massachusetts,
\end{abstract} USA,Tel 5087132183,Email majaz.moonis@umassmemorial.org

Received: August 12,2014 | Published: November 21, 2014
Abbreviations: BDNF, Brain Derived Neuro Trophic Factor; HGF, Hepatocyte Growth Factor; NMDA, N-Methyl-d-Aspartate; SVZ, Sub Ventricular Zone; DTI, Diffusion Tensor Imaging; DRS, Disability Rating Scale; MBI, Modified Barthel Index

\section{Introduction}

The injury, repair and recovery after stroke have been extensively defined. The first epoch is related to acute injury and takes place in the first initial hours after stroke when changes in blood flow, edema, metabolism rate and diaschisis occurs. A second epoch is related to repair, which starts days after stroke and lasts for several weeks and is referred to as endogenous repair suggesting a golden period for initiating restorative therapies. A third epoch occurs weeks to months after stroke when spontaneous recovery gains have plateaued and this represents a stable but modifiable early and late chronic phase.

The neuro embryology of human life is governed by the principle of ontogeny repeats phylogeny i.e., the injured cerebral tissue reverts to a quasi developmental state, where angiogenesis and synaptogenesis are evident which express proteins that aid brain remodeling. These processes remodel the brain and lead to an improved neurological function throughout the learning processes of human life.

The ultimate aim of any therapeutic strategy is maximum restoration possible and eventual return to normalcy of function. The non regenerating capacity of an injured adult brain has been challenged in the recent past and neural plasticity has been documented in both global and focal models of animal ischemia. ${ }^{1}$ the prospects of repairing an injured nervous system using putative restorative therapies seem promising.

Numerous pharmacological agents are under investigation since a decade which reflects developmental processes. Brain derived neuro trophic factor ${ }^{2}$ (BDNF), hepatocyte growth factor ${ }^{3}$ (HGF), granulocyte macrophage colony stimulating factor (FM-CSF) $)^{4,5}$ and other agents such as minocycline has proved to be efficacious in restoring neurofunctional benefits in preclinical and clinical studies.
Phosphodiesterase 5 inhibitors, statins and agents that increase high density lipoproteins and hormones that pioneered the use of thymosin beta 4 and carbamylated erythropoietin for the treatment of stroke and neural injury have been in use extensively. Chopp et al. ${ }^{6}$ published literature recently on the use of multifactor restorative agent cerebrolysin for stroke therapy.

\section{Pathophysiology of ischemic stroke ${ }^{7}$}

The degree and duration of impaired blood flow determines the extent and pattern of cerebrovascular damage. Ischemia causes loss of membrane potentials leading to anoxic depolarization and kills uniquely vulnerable neurons such as pyramidal neurons in the CA1 and CA4 zones of the hippocampus while sparing other neurons and glial cells. Deprivation of oxygen supply to the brain tissue leads to activation of the ischemic cascade with a series of molecular mechanisms being activated. There is depletion of adenosine triphosphate and consequent high levels of lactate and unbuffered hydrogen ions.

Failure of energy dependent mechanisms lead to deterioration of membrane ion gradients, opening of selective ion channels and equilibration of most intracellular and extracellular ions. Thus potassium ions leave the cell, sodium, chlorine and calcium enter and many excitatory neurotransmitters (glutamate, aspartate) are released in potentially toxic concentrations. The intracellular entry of calcium is made largely possible by the activation of two types of receptors, Voltage gated (L-type) and/or several N-methyl-d-aspartate (NMDA) and quisqualate $(\mathrm{Q})$ post synaptic receptor/channel complexes by glutamate. Agents that prevent white blood cells from adhering to vessel walls, limit formation of free radicals, or promote neuronal repair may protect the brain from additional injury during reperfusion. Neuroprotective agents that work primarily during reperfusion may have a longer window of therapeutic effect than drugs that work earlier in the ischemic cascade. 


\section{Agents for neuroprotection}

NMDA receptor modulation ${ }^{8}$ The most commonly studied neuroprotective agents for acute stroke which block N-methyl-daspartate (NMDA) receptors. Dextromethorphan, a noncompetitive NMDA antagonist was the first NMDA antagonist studied in human stroke patients with a large efficacy trial using GV150526 with 1367 patients completed in 2000. Although the drug was reported to be safe and well tolerated, no improvement was observed in any of the 3-month outcome measures. Magnesium is another agent with actions on the NMDA which reduce ischemic injury by increasing regional blood flow, antagonizing voltage-sensitive calcium channels and blocking the NMDA receptor with Magnesium Phase III (FASTMAG) Trial currently under run.

Non NMDA receptor modulation: Clomethiazole, a g-amino butyric acid agonist, decreases excitatory neurotransmission by increasing activity of inhibitory pathways. In Europe, clomethiazole's central nervous system inhibitory properties have been used for anticonvulsant and sedative effects. The potential efficacy of Clomethiazole as a neuroprotective agent in ischemia was first investigated in Europe as part of the Clomethiazole Acute Stroke Study. Patients received a 24-h IV infusion of Clomethiazole or placebo within $12 \mathrm{~h}$ of symptom onset. Nalmefene (Convene) is a narcotic receptor antagonist that reduces levels of excitatory neurotransmitters contributing to cellular injury in early ischemia. Post hoc analyses of early studies suggest that the drug may have more benefit in patients younger than 70 years. However, a later clinical trial in which the drug was administered intravenously (IV) within $6 \mathrm{~h}$ of symptom onset showed no benefit. The exact mechanism of action of lubeluzole, a drug effective in animal models, is unclear. The drug may block sodium channels in cells. In addition, it may reduce the release of nitric oxide, a neurotransmitter generated by activation of the NMDA receptors. Free radical agents like NXY-059 are under investigation in Stroke trials. ${ }^{9}$

Tetracycline derivatives: Minocycline: Minocycline is the second generation tetracycline derivative known to have anti-inflammatory effects independent of its antimicrobial action. Recent studies have shown that minocycline prevents microglial activation, and also has notable beneficial effects in animal models of global and transient focal cerebral ischemia and other brain injuries. ${ }^{10}$ The proposed mechanisms of minocycline include anti-inflammatory effects, reduction of microglial activation, MMP reduction, nitric oxide production and inhibition of apoptotic cell death. ${ }^{11}$ In a randomized single blinded study, Padma et al. ${ }^{12}$ studied the effects of oral Minocycline ( $200 \mathrm{mg} /$ day for 5 days) post stroke versus placebo. Out of 50 patients included in the trial, patients who received minocycline had significant improvement in stroke outcome as noted on NIHSS, $\mathrm{mBI}$ and $\mathrm{mRS}$ scores. Larger trials are needed to confirm the above findings.

\section{Agents for neurorestoration}

\section{Augmentation of nitric oxide cascade}

Nitric Oxide is an integral molecule involved with maintaining endothelial cell integrity, as well as participating in hemodynamic homeostasis. ${ }^{13} \mathrm{NO}$ is produced by a variety of cells, including vascular smooth muscle cells and neurons suggesting its role in neurogenesis by an increased expression of neuronal NO synthase within the sub ventricular zone (SVZ) during embryogenesis. It has been reported that treatment with NO 24 hours post stroke in rat models augmented neurogenesis and improvement in functional outcome despite no change in infarct volume. $\mathrm{NO}$ is also a potent activator of soluble guanylate cyclase, which converts GTP to cGMP. ${ }^{14}$ Delivery of NO donor increases cGMP levels in both ischemic and non-ischemic rats, suggesting a putative role of NO in neuro-angiogenesis and a downstream mediator of cGMP whose action is increased by inhibiting Phosphodiesterase 5 (PDE5) enzyme. The strategy of increasing the downstream mediator cGMP without affecting NO levels may be preferred due to the mixed outcomes in stroke reported in animal models following alterations in NO levels. A major PDE5 inhibitor is sildenafil. Animals treated with sildenafil post stroke achieved significant and substantial increase in neurological functional recovery. Phase I trials in humans with acute stroke are currently on going. An improved cerebral blood flow (CBF), neurogenesis and synaptogenesis following experimental stroke, even when therapy is with Sildenafil up to 1 caged animal is delayed. ${ }^{15}$ Studies have reported the improvements in functional outcomes occurred despite no change in infract volume, nevertheless these functional improvement will need to be demonstrated in clinical trials. Functional MRI or diffusion tensor imaging (DTI) may assist in answering these questions which demonstrate improvements in structure, organization and functional connectivity via acting as bio surrogate markers. Other agents such as statins, also greatly enhance neurological recovery post stroke. Drugs which increase high density lipoproteins (HDL) such as slow release niacin have also been employed to treat stroke and have shown substantial neurological benefit when treatment is initiated days after stroke. Other Neurorestorative agents under investigation are erythropoietin (EPO), carbamylated EPO (CEPO) and Thymosin B4.

Current physical rehabilitation therapies could be enhanced using pharmacological approach thereby helping to re establish lost neuronal connections. It has been elegantly established by Clarkson et al. ${ }^{16}$ that inhibiting tonic GABA (gamma amino butyric acid) signaling days improves locomotors function after stroke, suggesting that therapeutic approaches are less time sensitive than acute reperfusion therapies. P13k/Act and sonic hedgehog (Shh) pathways are activated by molecular and signal transduction genes which promote brain plasticity. Various pharmacological agents by means of signal transduction pathways can induce CNS plasticity that enhances functional recovery from stroke and neural injury.

\section{Neurotrophic agents and growth factors}

Basic fibroblast growth factor (FGF) was shown to protect against excitatory amino acid toxicity In vitro, basic FGF chimeric peptide was highly effective in reducing infarct volume in a rodent model of permanent focal ischemia. ${ }^{17} \mathrm{FGF}$ has been investigated in phase II/III trials. The results of the Clinical Safety Trial of Intravenous Basic Fibroblast Growth Factor in Acute Stroke did not report any serious adverse events. ${ }^{18}$ The European-Australian phase II/II safety and efficacy trials were terminated in the middle as no significant improvement was noted, although there was a trend towards treatment advantage. ${ }^{19}$

Recombinant erythropoietin (Epo) was reported to be safe and efficacious in a proof-of-concept study. ${ }^{20} \mathrm{~A}$ phase II/III study (522 patients) was negative and showed a higher death rate and complications in patients receiving Epo; possible interaction with rTPA was cited as a likely cause of increased mortality. ${ }^{21}$ Intravenous granulocyte colony stimulating factor (G-CSF) has also been investigated in a dose escalation phase II a study. ${ }^{22}$ (AXIS: 44 subjects, drug administered within 12 hours). The authors reported good tolerability and suggest further investigation.

Cerebrolysin, a peptide-based rug is another candidate with potential for approval to be used as a restorative agent. Multiple 
laboratories have demonstrated the safety and efficacy of this drug in the treatment of experimental stroke. Cerebrolysin, is another potential agent used as a restorative agent, which is a peptide-based rug used in multiple laboratories studying its safety and efficacy in the treatment of experimental stroke. This drug induces neurogenesis and angiogenesis in animal models of stroke and concomitantly enhances brain plasticity and recovery from stroke.

\section{Clinical trials on autologous bone marrow derived stem cells therapy in chronic stroke}

This unblinded, non randomized case control study dealt with the safety and efficacy of intravenous autologous bone marrow derived mononuclear and culture expanded mesenchymal stem cells in stroke. ${ }^{17}$ Adult patients were recruited with the inclusion criteria as: 3 months to 2 years after stroke, power of hand muscles of at least 2; Brunnstrom stage 2-5; NIHSS of 4-15, conscious and cooperative with assessments done for strength, tone (modified Ashworth), Fugl Meyer (FM) scale for upper limb, Edinburgh handedness inventory, modified Barthel Index (mBI) and functional MRI including DTI was performed at baseline, 8 and 24 weeks of stem cell infusion. Patients were screened and educated about stem cells and bone marrow aspiration technique prior to stem cell infusion. ${ }^{23}$ Forty stroke patients were recruited with the above inclusion criteria. Twenty were given stem cells followed by 8 weeks of physiotherapy, serving as experimental/stem cell group and 20 patients were administered physiotherapy regime alone. $50-60$ million cells in $250 \mathrm{ml}$ of saline was infused intravenously over 2-3 hours. The baseline clinical and radiological scores between the experimental and control groups were statistically insignificant. The safety profile was normal with no mortality or cell related adverse reactions in stem cell patients. On comparison between experimental and control groups, mBI was statistically significant on follow up at 24 weeks $(p=0.05)$. Laterality Index (LI) of BA 4 and BA 6 was insignificant at 8 and 24 weeks follow up, as also in the FA ratio, fiber length and fiber number ratio between the two groups. An increased number of cluster activation in Brodmann areas BA 4, BA 6 was observed post stem cell infusion indicating neural plasticity. The study was conclusive for safety and feasibility of intravenous autologous stem cell infusion. ${ }^{24,25}$ Stem cells may act as "scaffolds" for neural transplantation and may aid in repair mechanism. ${ }^{26}$

\section{Enhancing recovery with special reference to walking and aphasia after stroke}

Motor weakness and the inability to walk have been the primary targets for testing interventions that may improve after stroke. Physical therapeutic interventions enhance recovery after stroke; however, the timing, duration and type of intervention require clarification and further trials. Pharmacotherapy, in particular with dopaminergic and selective serotonin-reuptake inhibitors, shows promise in enhancing motor recovery after stroke; however, further large scale trials are required. ${ }^{27}$

The complex activity of walking requires the integration of sensory, visual, perceptual and cognitive inputs. ${ }^{28}$ Many varied strategies and techniques are undergoing assessment including pharmacological therapy for aphasia, transcranial magnetic stimulation for motor recovery and cognitive rehabilitation for attention deficits. ${ }^{29}$ It is possible that when used in combination these techniques may be symbiotic and synergistic. Much of the research in the area of stroke has focused on recovery of walking. Walking is a basic human function, often affected by stroke, more easily observed, more easily measured and potentially more easily rehabilitated than other functional deficits.$^{30}$ Besides loss of power in lower limb, walking also relies on the integrity of the trunk for balance, and the upper extremity for associated walking movements.

Currently the only pharmacological agent that has been shown to alter the natural history and recovery after stroke is tissue plasminogen activator given within 4.5 hours after stroke onset. However, a novel era in pharmacotherapeutics for stroke recovery may be in the horizon. Another study. ${ }^{31}$ randomized patients of severe traumatic brain injury (4-16 weeks) to amantadine (for 4 weeks), or placebo and then assessed at 6 weeks. It was observed that Amantadine, a dopamine agonist increased the speed of recovery during the active treatment phase. Disability Rating Scale (DRS) between baseline and at 6 weeks was similar in both groups, a post hoc analysis at the end of 4 weeks showed that more patients on amantadine had an improvement in their Disability Rating Scale scores.

Dopaminergic agents and selective serotonin-reuptake inhibitors (SSRIs) are known in the in altering the natural history of recovery after stroke. ${ }^{32}$ Dopamine is a neurotransmitter that may promote neuroplasticity in the cerebral cortex through schemas in brain, via memory and learning principles. ${ }^{33,34} \mathrm{~A}$ single oral dose of $100 \mathrm{mg}$ of levodopa and $25 \mathrm{mg}$ of carbidopa can enhance the ability of patients with chronic stroke to encode an elementary motor function. Scheidtmann et al. ${ }^{35}$ randomized patients ( 3 weeks and 6 months post stroke) to either 3 weeks of $100 \mathrm{mg}$ of levodopa with carbidopa or placebo daily. Patients receiving levodopa had significant improvement in motor recovery and independent ambulation. Subsequent small studies using levodopa with or without methylphenidate. ${ }^{36}$ or levodopa with or without amphetamine. ${ }^{37}$ could not show a difference in motor recovery or improvement in functional outcomes with treatment. An ongoing study which commenced in 2010, has enrolled 572 patients are to receive $100 \mathrm{mg}$ of levodopa and $25 \mathrm{mg}$ of carbidopa, or placebo, 1 hour before physiotherapy. The primary outcome will assess the number of patients walking independently at 8 weeks after randomization.

Animal studies suggest that SSRIs may be involved in neurogenesis and activation of cortical motor areas modulating neuronal plasticity .${ }^{38}$ These drugs are essential in maintaining sleep rhythm, and neurotransmitters levels within the brain and have been tried in stroke rehabilitation trials. A single dose of citalopram can normalize the balance in cortical excitability, as measured by transmagnetic stimulation. Patients more than 6 months after stroke, in a single dose cross over experiment with citalopram, showed improvement in hand dexterity as measured by the nine-hole peg test.$^{39} \mathrm{~A}$ single dose of fluoxetine given to patients, 2 to 3 weeks after stroke showed improved motor skills on the none-hole peg test, and increased activation of the affected side on functional resonance imaging. ${ }^{40}$

Chollet et al. ${ }^{41}$ randomized 118 acute ischemic stroke patients (5-10 days of event) into two arms: one group was administered with fluoxetine $(20 \mathrm{mg}$ /day by mouth) and another group placebo. Patients were assessed using the Fugl-Meyer motor scale (motor score varies from 0 to 100,66 points upper limb, 34 points lower limb; movements measured as none, partial or full) at the end of three months (90 days). The mean improvement in the total Fugl-Meyer motor scale and functional independence measure from baseline to 90 days was significantly higher in those patients treated with fluoxetine. A recent meta-analysis of randomized controlled trials that recruited stroke patients treated with an SSRI compared to usual care or sham identified 52 trials for analysis. The use of SSRIs is associated with an improvement in functionality, disability status, neurological impairment and depression; more large randomized trials are needed to derive definitive conclusions. ${ }^{42}$ 


\section{Amphetamines and motor rehabilitation}

Amphetamines are potent CNS stimulators of phenethylamine class which increase biogenic amines acting as excitatory neurotransmitter. Robust animal and human literature suggest that amphetamines coupled with physiotherapy aid in functional recovery via modulation of noradrenergic system. Brain is malleable to external and internal inputs, which has been credence through various reports. Changes in catecholaminergic, particularly nor epinephrine, functioning after brain injury have been correlated with changes in the rate of recovery after injury. ${ }^{43}$ Another trial reported that a single dose of AMPH given first day of treatment initiated 10 days after cortical infarct facilitated the rate of recovery (AMPH 68\%) compared to saline (27\%) treated animals. The cochraine review.$^{44}$ of amphetamines off late has suggested that 10 trials with 106 stroke patients underwent AMPH therapy and it was found that AM [PH did not prove to be beneficial in reducing mortality (Pero OR 1.5, 95\% CI- 0.6 to 3.3). Other 6 studies suggested that there was an evidence of a better relative change from baseline to follow up in motor function (WMD- 6.1 points, 95\% CI -10.4 to -1.9$)$. Schuster et al 2011 have recently reported increased performance in ADL and arm function in 8 patients who were administered with $10 \mathrm{mg}$ of AMPH per day ${ }^{45}$

\section{Neurorestorative therapy pharmacotherapy: Is there a hope?}

using

Is pharmacological restorative therapy post stroke merely a chimera? A perusal of clinical trials of neurorestorative agents certainly seems depressing at first glance. Nevertheless, if experimental evidence of neurorestoration is definite, why then has it not been replicated in clinical domains?

Translating restorative agents from bench to bed has to be performed with caution and care, as the failure is evidenced from clinical trials on several previous occasions. For eg; erythropoietin (EPO) demonstrated in potent therapeutic benefits in multiple clinical studies for the treatment of stroke, and appeared to be a strong candidate for translational research. The phase III clinical trial was terminated because of high mortality and adverse events. Of the stroke patients in the reported trial, $63.4 \%$ were administered rTPA, EPO was not tested in the laboratory in conjunction with rTPA. Another subsequent study clearly demonstrated adverse events in animals when treated with combination of EPO with rTPA which was observed in the clinical trial.

\section{Criticisms of animal studies include the following}

1) Small sample size (underpowered), 2) Lack of randomization, 3) Variable injury levels, 4) Inter-species variations, 5) Confounding variables (hypothermia, use of anesthetic agents), 6) Lack of evaluation of the dose-response relationship and side-effects (therapeutic index), 7) Inadequate outcome measures or biomarker end-points and 8) Flawed statistical analysis. On the basis of these observations, the Stroke Therapy Academic Industry Roundtable (STAIR) recommendations were developed for providing a stronger preclinical database for potential therapeutic agents.

\section{Biomarkers of restorative therapy induced stroke recovery}

A biomarker is an indicator of disease state that is useful clinically as a substitute measure, reflecting underlying molecular/cellular events that are difficult to measure directly ${ }^{46} \mathrm{~A}$ relevant biomarker for stroke recovery is the one that reflects a brain event relates to recovery and that correlates with behavioral state. Neurorestoration is a concept that has been proven emphatically in several experimental models of stroke. The lack of proof in clinical settings will continue to be discouraging until the reasons for failure in this endeavor are examined. The trials of the past cannot be termed as failures as they definitely have contributed to our understanding of the complex biology of brain injury. This knowledge must provide an impetus for the development of superior candidate molecules and methodological interventions that will enhance drug development as well as clinical testing.

\section{Acknowledgments}

None.

\section{Conflicts of interest}

None.

\section{References}

1. Shang J, Deguchi K, Ohta Y, et al. Strong neurogenesis, angiogenesis, synaptogenesis, and antifibrosis of hepatocyte growth factor in rats brain after transient middle cerebral artery occlusion. $J$ Neuroscie Res. 2011;89(1):86-95.

2. Chen J, Zhang C, Jiang H, et al. Atorvastatin induction or VEGF and BDNF promotes brain plasticity after stroke in mice. $J$ Cereb Blood $F$ Metab. 2005;25(2):281-290.

3. Doeppner TR, Kaltwasser B, Elali A, et al. Acute hepatocyte growth factor treatment induces long-term neuroprotection and stroke recovery via mechanisms involving neural precursor cell proliferation and differentiation. J Cereb Blood F Metab. 2011;31(5):1251-1256.

4. Navarro-Subrino M, Rosell A, Penalba A, et al. Role of endogenous granulocyte-macrophage colony stimulating factor following stroke and relationship toneurological outcome. Curr Neurovasc Res. 2009;6(4):246-251.

5. Sugiyama Y, Yagita Y, Oyama N, et al. Granulocyte colony-stimulating factor enhances arteriogenesis and ameliorates cerebral damage in a mouse model of ischemic stroke. Stroke. 2011;42(3):770 -775.

6. Harvey RL, Chopp M. The therapeutic effects of cellular therapy for functional recovery after brain injury. Phy Med Rehabil Clin N Am. 2003;14(1 supple):S143-151.

7. Onwuekwe IO, Adikaibe BE. Ischemic stroke and neuroprotection. Ann Med Health Sci Res. 2012;2(2):186-190.

8. Obrenovitch T, Sarna G, Symon L. Ionic homeostasis and neurotransmitter changes in ischemic. In: Krieglstein J, Oberpichler $\mathrm{H}$ (Eds.), Pharmacology of cerebral ischemic. Stuttgart, WVS, Germany. 1990;pp.97-112.

9. Choi DW. Ionic dependence of glutamate neurotoxicity. J Neurosci. 1987;7(2):369-379.

10. Fagan SC, Waller JL, Nichols FT, et al. Minocycline to improve neurologic outcome in stroke (minos): A dose-finding study. Stroke. 2010;41(10):2283-2287.

11. Murata Y, Rosell A, Scannevin RH, et al. Extension of the thrombolytic time window with minocycline in experimental stroke. Stroke. 2008;39(12):3372-3377.

12. Padma MV, Bhasin A, Bhatia R, et al. Efficacy of minocycline in acute ischemic stroke: A single-blinded, placebo-controlled trial. Neurol India. 201260(1):23-28.

13. Bredt DS, Snyder SH. Nitric Oxide: A physiologic messenger molecule. Anпu Rev Biochem. 199463:175-195.

14. Palmer RM, Ferrige AG, Mocada S. Nitric oxide release accounts for the biological activity of endothelium-derived relaxing factor. Nature. 1987327(6122):524-526. 
15. Zhang RL, Zhang Z, Zhang L, et al. Delayed treatment with sildenafil enhances neurogenesis and improves functional recovery in aged rats after focal cerebral ischemia. J Neurosci Res. 2006;83(7):1213-1219.

16. Clarkson AN, Huang BS, Macisaac SE, et al. Reducing excessive $G A B A$-mediated tonic inhibition promotes functional recovery after stroke. Nature. 2010;468(7321):305-309.

17. Ay H, Ay I, Koroshetz WJ, et al. Potential usefulness of basic fibroblast growth factor as a treatment for stroke. Cerebrovasc Dis. 19999(3):131135

18. The FIBLAST Safety Study Group. Clinical safety of intravenous BFGF in acute stroke. Stroke. 199829:287.

19. Bogousslavsky J, Victor SJ, Salinas EO, et al. European-Australian Fiblast (Trafermin) in acute stroke: results of the European-Australian phase II/III safety and efficacy trial. Cerebrovasc Dis. 2002;14(3 4):239-251

20. Ehrenreich H, Hasselblatt M, Dembowski C, et al. Erythropoietin therapy for acute stroke is both safe and beneficial. Mol Med. 2002;8(8):495-505.

21. Ehrenreich H, Weissenborn K, Prange H, et al. EPO Stroke Trial Group Recombinant human erythropoietin in the treatment of acute ischemic stroke. Stroke. 2009;40(12):e647-56.

22. Schabitz WR, Laage R, Vogt G, et al. AXIS: A trial of intravenous granulocyte colony stimulating factor in acute ischemic stroke. Stroke. 2010;41(11):2545-2551.

23. Bhasin A, Srivastava MV, Mohanty S, et al. Stem cell therapy. A clinical trial in Stroke. Clin Neurol Neurosurg. 2013;115(7):1003-1008.

24. Bhasin A, Srivastava M, Bhatia R, et al. Autologous intravenous mononumclear stem cell therapy in chronic ischemic stroke. J Stem Cells Regen Med. 2012;8(3):181-189.

25. Bhasin A, Srivastava MV, Kumaran SS, et al. Autologous mesenchymal stem cell therapy in chronic stroke. Cerebrovasc Dis Extra. 2011;1(1):93-104

26. Bhasin A, Srivastava MV, Bhatia R, et al. Review- Stem cell in Neurological Disorders; Indian perspective. J Stem Cell Res and Thera (epub ahead of print). 2014

27. Greener J, Enderby P, Whurr R. Pharmacological treatment for aphasia following stroke. Cochrane Database Syst Rev. 2001 ;(4):CD000424.

28. Rosser N, Floel A. A pharmacological enhancement of motor recovery in subacute and chronic stroke. NeuroRehabilitation. 2008;23(1):95-103.

29. Hsu WY, Cheng CH, Liao KK, et al. Effects of repetitive Transcranial Magnetic Stimulation on Motor Functions in Patients with Stroke: $A$ meta-analysis. Stroke. 201243(7):1849-1857.

30. Langhorne P, Bernhardt J, Kwakkel G. Stroke rehabilitation. Lancet. 2011377(9778):1693-1702.

31. Giacino JT, Whyte J, Bagiella E, et al. Placebo-controlled trial of amantadine for severe traumatic brain injury. $N$ Engl J Med. 2012;366(9):819-826.
32. Gu Q. Neuromodulatory transmitter systems in the cortex and their role in cortical plasticity. Neuroscience. 2002111(4):815-835.

33. Berends HI, Nijlant JM, Movig KL, Van Putten MJ, et al. The clinical use of drugs influencing neurotransmitters in the brain to promote motor recovery after stroke: a Cochrane systematic review. Eur J Phys Rehabil Med. 2009;45(4):621-630.

34. Floel A, Hummel F, Breitenstein C, et al. Dopaminerigic effects on encoding of a motor memory in chronic stroke. Neurology. 2005;65(3):472-474

35. Scheidtmann K, Fries W, Müller F, et al. Effect of levodopa in combination with physiotherapy on functional motor recovery after stroke: a prospective, randomized, double-blind study. Lancet. 2001;358(9284):787-790

36. Lokk J, Salman Roghani R, Delbari A. Effect of methylphenidate and/or levodopa coupled with physiotherapy on functional and motor recovery after stroke- a randomized, double-blind, placebo-controlled trial. Acta Neurol Scand. 2011;123(4):266-273

37. Sonde L, Lokk J. Effects of amphetamine and/or 1-dopa and physiotherapy after stroke - a blinded randomized study. Acta Neurol Scand. 2007;115(1):55-59.

38. Acler M, Robol E, Fiaschi A, et al. A double blind placebo RCT to investigate the effects of serotonergic modulation on brain excitability and motor recovery in stroke patients. J Neurol. 2009;256(7):1152-1158.

39. Zittel S, Weiller C, Liepert J. Citalopram improves dexterity in chronic stroke patients. Neurrehabil Neural Repair. 2008;22(3):311-314.

40. Pariente J, Loubinoux I, Carel C, et al. Floouxetine modulates motor performance and cerebral activation of patients recovering from stroke. Ann Neurol. 2001;50(6):718-729.

41. Chollet F, Tardy J, Albucher JF, et al. Flouxetine for motor recovery after acute ischemic stroke (FLAME): a randomized, placebo -controlled trial. Lancet Neurol. 2001;10(2):123-130.

42. Mead GE, Hsieh CF, Lee R, et al. Selective serotonin reuptake inhibitors (SSRIs) for stroke recovery. Cochrane Database Syst Rev. 2012;310(10):1066-1067.

43. Walker B Amphetamines and post stroke rehabilitation: indications and controversies. Euro J Phy Rehab Med. 2012;49(2):251-260.

44. Martinsson L, Hardemark HG, Esborg S Amphetamines for improving recovery after Stroke. Cochraine Database Syst Rev. 2008;(1):CD002090.

45. Schuster C, Maunz G, Lutz K, et al. Dexamphetamine improves upper extremity outcomes during rehabilitation after stroke-a pilot randomized controlled trial. Neurorehabil Neural repair. 2011;25(8):749-755.

46. Burke E, Cramer SC Biomarkers and predictors of restorative therapy effects after stroke. Curr Neurol Neurosci Rep. 2013;13(2):329. 